\title{
Subjektive Nebenwirkungsklagen von Borderline-Patienten - zwei Jahre nach einer tagesklinischen Behandlung
}

\author{
Christoph Richter ${ }^{a, b}$ \\ ${ }^{a}$ Klinik für Psychiatrie und Psychotherapie, Charité - Universitätsmedizin Berlin, Campus Mitte, Berlin, Deutschland; \\ ${ }^{\text {b}}$ Klinik für Psychiatrie, Psychotherapie und Psychosomatik, Vivantes Klinikum Kaulsdorf, Berlin, Deutschland
}

\section{Schlüsselwörter \\ Borderline-Persönlichkeitsstörung · Dialektisch- \\ behaviorale Therapie · Negative Effekte · Prädiktoren · \\ Psychotherapieforschung $\cdot$ Tagesklinik}

\section{Zusammenfassung}

Einleitung: Die Erfassung von negativen Effekten ( $\mathrm{nE}$ ) von Psychotherapie (PT) ist wichtig, um Schwachstellen von PT zu identifizieren und die Behandlungsqualität zu verbessern. Studienziel ist, zu untersuchen, welche nE Patienten nach einer tagesklinischen Behandlung für Borderline-Persönlichkeitsstörungen (BPS) mit einem Dialektisch-Behavioralen Therapieschwerpunkt (DBT) beschreiben, welche Einflussfaktoren vorliegen und wie die Klagen inhaltlich zu verstehen sind. Material und Methoden: Katamnestisch wurden BPS-Patienten 22,6 Monate nach Therapieende befragt, an welche $n E$ sie sich mit Bezug zu einer zwölfwöchigen tagesklinischen Behandlung erinnern. Messinstrumente waren BDI, BSL-95 und INEP. Ergebnisse: 35 Frauen und 6 Männer antworteten (50\% von 82). Im Mittel wurden 11,2 nE benannt, die subjektiv auf die DBT-Behandlung zurückgeführt wurden. 97,6\% berichten von Verschlechterung ihres Befindens, 87,8\% erinnern sich an Probleme in der therapeutischen Beziehung, 43,9\% klagten über Verschlechterungen in der Beziehung, 70,7\% über finanzielle Probleme in Folge der tagesklinischen Behandlung und 29,3\% über Stigmatisierungserleben. Die Zahl der nE korrelierte mit höherem Alter zum Therapiestart $(p<0,01)$ sowie höherer Symptomlast anhand der BSL-95 ( $p<0,01)$ bzw. BDI $(p<0,01)$ zum Befragungszeitpunkt. Diskussion: Es konnte gezeigt werden, dass Patienten bei Vorlage des INEP über eine erheb- liche Rate an Negativerfahrungen im Rahmen der tagesklinischen Behandlung klagen. Der lange Rückerinnerungszeitraum von 3 bis 51 Monaten und die Korrelation mit der aktuellen Symptomatik stellen die Validität der Antwort in Frage. Ergebnisse von Selbstratinginstrumenten zur Nebenwirkungserfassung bedürfen einer sehr sorgfältigen Interpretation.

(C) 2020 The Author(s) Published by S. Karger AG, Basel

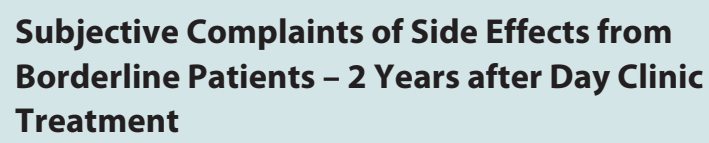

\section{Abstract}

Introduction: The recording of negative effects $(\mathrm{nE})$ of psychotherapy (PT) is important in order to identify weak points of PT and to improve the quality of treatment. The aim of the study is to find out which $\mathrm{nE}$ patients describe after day-clinic treatment for borderline personality disorders (BPD) with a dialectical behavioral therapy (DBT), which influencing factors are present, and how the content of the complaints is to be understood. Material and Methods: 22.6 months after the end of therapy, BPS patients were asked catamnestically which $\mathrm{nE}$ they remembered in relation to a 12-week day-clinic treatment. Mea-
(C) 2020 The Author(s)

Published by S. Karger AG, Basel

This is an Open Access article licensed under the Creative Common Attribution-NonCommercial-4.0 International License (CC BY-NC) (http://www.karger.com/Services/OpenAccessLicense), applicable to the online version of the article only. Usage and distribution for commercial purposes requires written permission. 
suring instruments were BDI, BSL-95, and INEP. Results: 35 women and 6 men answered (50\% of 82). A mean of 11.2 nE was named, which was subjectively attributed to the DBT treatment. $97.6 \%$ report worsening of their condition, $87.8 \%$ recall problems in the therapeutic relationship, $43.9 \%$ report deterioration in their relationship, $70.7 \%$ report financial problems as a result of day-clinic treatment, and $29.3 \%$ about experience of stigmatization. The number of $\mathrm{nE}$ correlated with older age at the start of therapy $(p<0.01)$ and higher symptom burden based on the BSL-95 $(p<0.01)$ and BDI $(p<0.01)$ at the time of the survey. Discussion: It could be shown that when using the INEP, patients complain of considerable rates of negative experiences in the context of day-clinic treatment. The long recall period of 3 to 51 months and the correlation with the current symptoms call the validity of the answer into question. Results of self-rating instruments for recording side effects require very careful interpretation.

(c) 2020 The Author(s) Published by S. Karger AG, Basel

\section{Einführung}

Obwohl die Borderline-Persönlichkeitsstörung (BPS) nicht die häufigste psychische Erkrankung ist, bestimmt sie in Klinik und Praxis aber oft den Alltag und erschwert erwünschte Therapieerfolge [Crowell et al., 2009; Carpenter und Trull, 2013; Ellison et al., 2018]. Spezifische medikamentöse Therapieansätze gibt es derzeit nicht. Vielfältige Substanzklassen finden jedoch außerhalb der Zulassung symptombezogen und oft in Polypharmazie Anwendung [Sansone und Wiederman, 2009; Lieb et al., 2010].

Psychotherapeutische Ansätze sind für die Behandlung der BPS erfolgsversprechender [Stoffers et al., 2012]. Die Dialektisch-Behaviorale Therapie (DBT) ist eine störungsspezifische Therapie, deren Effektivität und Effizienz durch vielfältige kontrollierte Studien nachgewiesen wurde [Bohus und Kröger, 2011; Pasieczny und Connor, 2011; Stoffers et al., 2012; Cristea et al., 2017]. In Deutschland hat sich historisch die DBT im (teil)stationären Sektor zur am weitverbreitetsten störungsspezifisch psychotherapeutischen Methode der BPS entwickelt. Bisherige Studien zur Wirksamkeit der DBT zeigen erwünschte und nachhaltige Effekte konkret hinsichtlich der Abnahme von Suizidalität, Selbstverletzung und Klinikeinweisung [Kienast et al., 2014; Stiglmayr et al., 2014] als auch Kosteneffizienz [Levant et al., 2006; Kliem et al., 2010; Priebe et al., 2012; van den Bosch et al., 2014].

Im Vergleich dazu ist das Forschungsinteresse hinsichtlich unerwünschter Wirkungen oder negativer Effekte von Psychotherapie eher gering. So hat bisher Patientensicherheit für die psychotherapeutische Forschung keine Priorität [Duggan et al., 2014; Parry et al., 2016; Scott und Young, 2016].
Im Gegensatz dazu werden medikamentöse Therapien in allen Stadien ihrer Entwicklung diesbezüglich sorgfältig geprüft. Der Nachweis eines guten Risiko-NutzenVerhältnisses ist die Entscheidungsgrundlage für deren Zulassung durch die Food and Drug Administration (FDA) oder die European Medicines Agency (EMA). Psychotherapie unterliegt keiner solchen strengen Regulierung [Nutt und Sharpe, 2008]. Für medikamentöse Therapien konnte ein hoher Standard für ein Risiko-Nutzen-Verhältnis in der allgemeinen Aufmerksamkeit nicht zuletzt durch den Gesetzgeber, Forscher, Praktiker, Epidemiologen und Patienten erreicht werden.

Informationen über eventuelle Schäden oder Nebenwirkungen von Psychotherapie werden selbst im Forschungsbereich bisher nicht systematisch erfasst [Vaughan et al., 2014]. In Abschlussberichten psychologischer Studien werden unerwünschte Ereignisse, anders als in medikamentösen, selten dokumentiert [Duggan et al., 2014]. Damit ist unklar, in welchem Umfang psychotherapeutische Methoden, die für Patienten von Nutzen sind, auch unerwünschte Nebenwirkungen verursachen können [Petry et al., 2008; Freyberger et al., 2013; Jonsson et al., 2014; Vaughan et al., 2014; Holsting et al., 2017].

Als mögliche Ursachen dieser geringen Aufmerksamkeit werden Schwierigkeiten bei der Definition, ein möglicher Publikationsbias oder eine schlechtere theoretische Konzeptualisierung und methodische Probleme bei deren Untersuchung diskutiert [Mohr, 1995; Jacobi, 2001; Duggan et al., 2014].

Linden [2013; Freyberger et al., 2013] schlägt deshalb eine Systematik vor, wie Nebenwirkungen in der Psychotherapie definiert werden könnten. Hierbei sollten $\mathrm{Ne}$ benwirkung, Misserfolge, Abbrüche, Non-Responder, Rückfälle, Verschlechterungen, falsche Therapie, fehlerhaft angewandte Therapie, Ausbeutung und Missbrauch von Patienten, unerwünschte Ereignisse, negative Therapiewirkungen, Kunstfehler und Kontraindikationen voneinander unterschieden werden.

Erst in den letzten Jahren begann auch das Interesse, Erfassungsinstrumente negativer Effekte von Psychotherapie zu entwickeln. Als einer der ersten gilt die Vanderbilt Negative Indicators Scale [Suh et al., 1986]. Einige weitere Fragebögen wurden in der Zwischenzeit vorgeschlagen, etwa der Experiences of Therapy Questionnaire (ETQ) [Parker et al., 2013], das Inventar zur Erfassung negativer Effekte von Psychotherapie [Ladwig et al., 2014], der Fragebogen Nebenwirkungen stationärer Psychotherapie (NESTAP) [Brakemeier et al., 2012] und die von Linden entwickelte Checkliste unerwünschter Ereignisse (UE-ATR) [Linden, 2013]. Ebenfalls von Linden und Kollegen entwickelt wurde die Skala zur Erfassung unerwünschter Ereignisse in Gruppentherapien (UE-G) [Linden et al., 2015]. Ein weiteres Instrument ist das Inventar zur Beurteilung negativer Auswirkungen von Psy- 
chotherapie (INEP) [Ladwig et al., 2014]. Das INEP dient zur Feststellung ungünstiger Veränderungen durch Psychotherapie aus Patientensicht, ist praktikabel und wurde in mehreren Studien angewandt [Grüneberger et al., 2017; Abeling et al., 2018]. Auch gibt es vereinzelt größere naturalistische Studien im Kliniksetting zur Beschreibung negativer Effekte in Gruppentherapien [Schneibel et al., 2017].

Bei der Erfassung von unerwünschten Ereignissen gilt auch, dass Globalangaben wenig nützlich sind, sondern methoden- und bereichsspezifische Daten benötigt werden. Ein Beispiel sind unerwünschte Ereignisse bei DBT der BPS in einem tagesklinischen Setting, wozu es einige Berichte zur Symptomverbesserung gibt [Richter et al., 2014b; Richter, 2019], sich jedoch trotz umfangreicher Recherche keine Studien über mögliche negative Effekte finden ließen. Dies wäre aber von praktischer Bedeutung, da die Therapie der BPS allgemein besonders hohe therapeutische Anforderungen stellt u.a. durch deren komplexe und vielfältige Probleme in der sozialer Interaktion [Schmahl et al., 2014; Bohus, 2019; Signer et al., 2019]. Als Beispiel eines definierten Therapiesettings stellt tagesklinische Behandlung einen Mix aus ambulanter und stationärer Therapie dar. Diese ist alltagsnäher, mit weniger Regression und Hospitalismus behaftet als eine vollstationäre Therapie. Sie führt seltener zu einem Herausfallen aus sozialen Netzen und bietet eine große Bandbreite verschiedener therapeutischer Interventionen an [Hopf, 2018].

Die Frage ist, welche negativen Effekte unter diesen Rahmenbedingungen auftreten. Es wäre interessant, auch Einflussfaktoren zu untersuchen, die das Auftreten von unerwünschten Ereignissen moderieren, sei es auf Seiten der Patienten, Therapeuten oder der Umwelt.

Auf diesem Hintergrund sollte in einer katamnestischen Befragung explorativ untersucht werden, ob, welche und wie häufig negative Effekte aus Sicht von BPSPatienten nach einem zwölfwöchigen, tagesklinischen DBT-Programm berichtet werden und welche Einflussfaktoren es gibt.

\section{Methodik}

Eine tagesklinische Behandlung steht zwischen einer ambulanten und stationären Therapie. Die ambulante Behandlung hat Vorrang vor der stationären Behandlung, wenn das Behandlungsziel zweckmäßig und ohne Nachteil für die Patientin oder den $\mathrm{Pa}$ tienten mit den Mitteln der ambulanten Versorgung erreicht werden kann ( $\$ 39$ Absatz 1 Satz 2 SGB V). Die Aufnahme der Patienten in die hier untersuchte Tagesklinik erfolgt nach ärztlicher Indikationsprüfung mit ambulanter fachärztlicher Einweisung. Im Vorfeld war die ambulante Versorgung nicht mehr als ausreichend eingeschätzt worden. Gleichzeitig war das Interesse groß, weniger aus den sozialen Bezügen zu fallen und auch zu Hause zu wohnen, was bei einer vollstationären Therapieoption nicht mög- lich gewesen wäre. Teilweise lagen Empfehlungen von Akutkliniken vor, Direktverlegungen erfolgten jedoch nicht.

Die hier beispielhaft verwandte Tagesklinik 1 im Berliner Vivantes Wenckebach-Klinikum ist nach den Richtlinien des Dachverbands DBT zertifiziert. Entsprechend werden folgende Therapiekomponenten angeboten: DBT-Gruppen (Skillstraining, zwei Stunden pro Woche: Stresstoleranz, Emotionsregulation, soziale Kompetenz etc.), Einzeltherapie (1 h/Woche), Co-TherapeutenEinzel (praktische Umsetzung von Skills und DBT-Inhalten, $1 \mathrm{~h} /$ Woche), Psychoedukation (Basisgruppe 1,5 h/Woche), Bezugsgruppe (Peer Group, ca. 3 h/Woche), Achtsamkeitsgruppe (1 h/ Woche), Körpertherapie (Gruppe und/oder individuell, 1-2 h/ Woche), Teambesprechung/-Supervision für Therapeuten $(2 \mathrm{~h} /$ Woche) sowie die zweiwöchentliche Oberarztvisite. Die DBTGruppen (Skillstraining), Einzeltherapie und Teambesprechungen/-Supervision erfolgten nach dem DBT-Manual von Linehan [2016]. Acht Stunden Teamschulung pro Jahr werden durch einen anerkannten externen DBT-Supervisor erbracht. Zusätzlich findet vor allem über 4-5 Stunden pro Woche interaktionelle Ergotherapie als Kochgruppe statt. Außerdem sind etwa zwei Stunden für Vor- und Nachbearbeitung der Therapie (Hausaufgaben, eigenständiges Skillstraining) für die Patienten reserviert. Somit stehen direkt dem Patienten mindestens 15 Stunden Therapie über zwölf Wochen (insgesamt also etwa 180 Therapiestunden zur Verfügung). Dies ist um 100 Stunden mehr als in einem ambulanten Setting in Deutschland zumeist möglich ist.

Bei einem zur Zertifizierung nachzuweisenden Personalschlüssel von über $80 \%$ nach der Verordnung über Maßstäbe und Grundsätze für den Personalbedarf in der stationären Psychiatrie stehen zwei ausgebildete DBT-Therapeuten (ein Facharzt für Psychiatrie und Psychotherapie und eine approbierte Diplom-Psychologin), eine psychologische DBT-Therapeutin in Ausbildung und mindestens zwei DBT-Pflegekräfte/Fachtherapeuten zur Verfügung. Durch regelmäßige nachzuweisende Voraussetzungen für die DBT-Re-Zertifizierungen wird die Adhärenz klassischer DBTTechniken kontinuierlich beachtet [Lynch et al., 2007; McCay et al., 2016].

Ein Drittel der insgesamt 20 Behandlungsplätze ist für die Therapie von BPS-Patienten reserviert. Eine getrennte ca. zweiwöchige Diagnostikphase im Vorfeld wird unabhängig von der zwölfwöchigen Therapiedauer vorgehalten. Die anderen Krankheitsbilder stellen affektive und Angsterkrankungen, welche ein eigenständiges von der zertifizierten DBT unterschiedenes Therapieprogramm erhalten. Von Montag bis Freitag (8.30-16.00 Uhr) wird das DBT-Programm in dieser Tagesklinik angeboten. Am Wochenende ist die Tagesklinik geschlossen, eine Notversorgung erfolgt durch die Klinik [Richter et al., 2014a].

Diese Tagesklinik mit DBT-Modul bietet hiermit also die wesentlichen Elemente eines therapeutischen Milieus an: Diagnosebestätigung, intensive Therapie, einen therapeutischen Schutzraum, die Möglichkeit der Tagesstrukturierung, ein Übungsfeld, kontinuierliche Motivationsarbeit, Modelllernen vom Therapeuten und von Mitpatienten sowie soziale funktionalere Kontaktmöglichkeiten [Linden, 2014].

Im Rahmen einer retrospektiven Katamnese wurden 82 Patienten angeschrieben, die volljährig waren, das beschriebene DBTProgramm durchliefen (auch Einschluss vorzeitiger Abbrüche) und eine BPS mit mindestens 5 Kriterien nach DSM-IV mittels SKID-II zeigten. Per Brief wurden die ehemaligen Patientinnen und Patienten nach beendeter Therapie über den Zweck der Umfrage, die Pseudonymisierung und die Sicherheit der Datenverarbeitung informiert. Alle Antwortenden erklärten ihre Einwilligung. Die Freiwilligkeit war im Rahmen dieser retrospektiven Untersuchung explizit benannt worden. Ihnen wurde zugesichert, dass sie keine Nachteile bei möglichen zukünftigen Wiederauf- 
Tabelle 1. Soziodemographische Parameter

a Schulabschlüsse der Stichprobe zum Therapiezeitpunkt

\begin{tabular}{lrr}
\hline Schulabschluss & $n$ & Prozent \\
\hline Ohne & 2 & $4,9 \%$ \\
(Erw.) Hauptschulabschluss & 4 & $9,8 \%$ \\
Realschulabschluss & 17 & $41,5 \%$ \\
Abitur & 13 & $31,7 \%$ \\
Keine Angaben & 5 & $12,2 \%$ \\
\hline
\end{tabular}

b Allgemeine Parameter zur Stichprobenbeschreibung mit Korrelation zum INEP

\begin{tabular}{|c|c|c|c|c|c|}
\hline & $n=41(100 \%)$ & M & $\mathrm{R}$ & SD & $\begin{array}{l}\text { Korrelation mit } \\
\text { INEP-Gesamtanzahl }\end{array}$ \\
\hline Zeitraum seit Therapie bis zur Befragung (Monate) & $41(100 \%)$ & 21,6 & $3-51$ & 14,5 & $r=-0,08$ \\
\hline Therapiezeitraum (Tage) & $41(100 \%)$ & 83,2 & $21-128$ & 18,7 & $r=-0,03$ \\
\hline Alter ${ }^{\mathrm{a}}$ (Jahre) & $41(100 \%)$ & 32,9 & $19-51$ & 8,45 & $r=0,5^{* *}$ \\
\hline Anzahl DSM-IV-Kriterien ${ }^{\mathrm{a}}$ & $41(100 \%)$ & 6,3 & $5-9$ & 1,1 & $r=0,13$ \\
\hline Mindestanzahl Suizidversuche ${ }^{\mathrm{a}}$ & $34(82,9 \%)$ & 1,7 & $0-20$ & 3,5 & $r=-0,1$ \\
\hline $\mathrm{BDI}^{\mathrm{b}}$ (absolut) & $37(90,2 \%)$ & 24,3 & $4-42$ & 10,5 & $r=0,24^{* *}$ \\
\hline BSL-95' (absolut) & $36(87 \%)$ & 156,2 & $12-327$ & 75,3 & $r=0,31^{* *}$ \\
\hline
\end{tabular}

${ }^{\mathrm{a}}$ zum Therapiezeitpunkt, ${ }^{\mathrm{b}}$ zum Befragungszeitpunkt. ${ }^{*} p<0,05,{ }^{* *} p<0,01$. M, Mittelwert; R, Range; SD, Standardabweichung; $r$, Korrelation nach Pearson.

nahmen zu befürchten haben. Auch wurde darauf hingewiesen, dass die Befragung keine Fortsetzung einer Therapie ist. Der Datenschutzbeauftragte des kommunalen Vivantes Konzerns war intensiv mit einbezogen worden. Die Kriterien der Helsinki-Erklärung in der aktuellen Fassung wurden ebenfalls beachtet. Die Datensätze wurden pseudonymisiert, eine individuelle Zuordnung ist somit nicht herstellbar.

\section{Messinstrumente}

Das INEP enthält 21 Fragen, die vom Patienten selbst beantwortet werden, und ist unterteilt in "intrapersonelle Symptomänderungen", "Stigmatisierung", "Veränderungen im Bereich Partnerschaft, Freunde, Familie", "finanzielle und rechtliche Nachteile", "therapeutische Beziehung" und "therapeutisches Fehlverhalten”. Die Patienten können angeben, ob die Änderungen durch die Behandlung oder durch andere Umstände oder beides verursacht werden. Der Schweregrad wird im ersten Teil (A) des INEP zwischen +3 und -3 und im zweiten Teil $(B, C)$ subjektiv von 0 bis 3 skaliert [Ladwig et al., 2014]. "Unethisches Verhalten" als therapeutisches Fehlverhalten ist kein negativer Effekt, der auf die Psychotherapie an sich zurückzuführen ist. Daher wurden drei Punkte der Skala separat bewertet. Diese Art der Bewertung wurde von den Autoren des INEP [Ladwig et al., 2014] vorgeschlagen und auch in einer anderen Studie [Grüneberger et al., 2017] übernommen. Der Summenscore und die Häufigkeitsverteilung ermöglichen eine Beurteilung der Belastung negativer Effekte und einen Vergleich mit weiteren Studien.

Zur Erfassung der Symptomschwere wurde das Beck-Depressions-Inventar (BDI) [Hautzinger et al., 1995] sowie die Borderline-Symptom-Skala 95 (BSL-95) [Bohus et al., 2007] eingesetzt. Daten des BDI und der BSL-95 lagen zusätzlich für den Zeitraum zum eigentlichen Therapiestart (T1) und zum Therapieende (T2) aus einer Vorstudie tagesklinischer DBT vor [Richter et al., 2014a].
Zusätzlich wurde die Anzahl bzw. Quote der die Therapie Abbrechenden ermittelt.

Folgende patientenbezogene Charakteristika sollten als mögliche Einflussfaktoren negativer Effekte untersucht werden: Alter, Geschlecht, Schulabschluss, Ausprägung der BPS anhand von DSM-IV-Kriterien, BSL-95 und BDI sowie die Anzahl von Suizidversuchen. Diese Parameter lagen im Rahmen der strukturierten Anamneseerhebung und Quantifizierung der Symptomstärke für den gesamten Therapieprozess vor. Mögliche andere einflussnehmende Parameter sollen überprüft werden: Abbruchrate, Therapiezeitraum und Zeit nach dem Therapieende bis zur Befragung.

\section{Ergebnisse}

\section{Allgemeine Stichprobenbeschreibung}

Die Nachbefragung erfolgte im Durchschnitt 21,6 (Range 3-51, SD 14,5) Monate nach Entlassung aus der tagesklinischen Behandlung. Es antworteten 41 (50\%) von 82 Angeschriebenen, 35 Frauen $(85,4 \%)$ und 6 Männer $(14,6 \%) ; 31,7 \%$ hatten das Abitur, 4,9\% keinen Schulabschluss. Fünf (12,2\%) von 41 Antwortenden hatten die Therapie vorzeitig abgebrochen. In Tabelle 1 sind Altersverteilung, Therapiezeitraum tagesklinischer DBT, Zeit seit Therapieende bis zur Befragung, Anzahl erfüllter DSM-IV-Kriterien sowie Mindestanzahl an Suizidversuchen bis zum Therapiestart, als auch die Ausprägung des BDI und der BSL-95 dargestellt. 
Tabelle 2. Differenzierte Darstellung einzelner negativer Effekte und Bereiche anhand des INEP (ohne therapeutisches Fehlverhalten)

\begin{tabular}{|c|c|c|c|c|}
\hline INEP-Bereiche und Einzeleffekte & $n=41(100 \%)$ & M & $\mathrm{R}$ & $\mathrm{SD}$ \\
\hline Intrapersonelle Symptomatik & $40(97,6 \%)$ & 5,3 & $1-11$ & 2,9 \\
\hline Längere Phasen in schlechter psychischer Verfassung & $40(97,6 \%)$ & 2,1 & $1-3$ & 0,8 \\
\hline Erhöhte Belastung durch Ereignisse aus der Vergangenheit & $11(26,8 \%)$ & 2,3 & $1-3$ & 0,6 \\
\hline Veränderung als Mensch zum Negativen & $12(29,3 \%)$ & 1,1 & $1-2$ & 0,3 \\
\hline Erstmals Suizidgedanken durch die Therapie & $8(19,5 \%)$ & 1,7 & $1-3$ & 0,9 \\
\hline Verschlechterung der Fähigkeit, anderen zu vertrauen & $11(26,8 \%)$ & 1,6 & $1-3$ & 0,8 \\
\hline Verschlechterung des Befindens im Vergleich zu vor der Therapie & $7(17,1 \%)$ & 1,9 & $1-3$ & 0,9 \\
\hline Therapeutische Beziehung & $36(87,8 \%)$ & 3,8 & $1-12$ & 2,4 \\
\hline Verletztheit durch Aussagen des Therapeuten & $23(56,1 \%)$ & 1,9 & $1-3$ & 0,9 \\
\hline Schwierigkeiten, Entscheidungen allein zu treffen & $29(70,7 \%)$ & 1,7 & $1-3$ & 0,7 \\
\hline Abhängigkeit vom Therapeuten & $15(36,6 \%)$ & 1,5 & $1-3$ & 0,7 \\
\hline Gefühl, der Therapeut mache sich lustig über den Patienten & $8(19,5 \%)$ & 1,5 & $1-3$ & 0,9 \\
\hline Empfundener Zwang zu Interventionen (Konfrontationen, Rollenspiele, usw.) & $4(9,8 \%)$ & 2,5 & $2-3$ & 0,6 \\
\hline Partnerschaft, Familie, Freunde & $18(43,9 \%)$ & 3,3 & $1-7$ & 2,1 \\
\hline Zunahme von Konflikten in der Partnerschaft & $7(17,1 \%)$ & 1,9 & $1-3$ & 0,7 \\
\hline Verschlechterung des Verhältnisses zur Familie & $11(26,8 \%)$ & 2,1 & $1-3$ & 0,8 \\
\hline Verschlechterung des Verhältnisses zu Freunden & $10(24,4 \%)$ & 1,8 & $1-3$ & 0,8 \\
\hline Eifersucht des Partners auf die therapeutische Beziehung & $2(2,4 \%)$ & 2,5 & $2-3$ & 0,7 \\
\hline Finanzielle und rechtliche Nachteile & $29(70,7 \%)$ & 4,7 & 1-9 & 1,8 \\
\hline Probleme mit Versicherungen bzw. Angst davor & $10(24,4 \%)$ & 1,7 & $1-3$ & 0,7 \\
\hline Mehr finanzielle Sorgen & $28(68,3 \%)$ & 2,1 & $1-3$ & 0,8 \\
\hline \multicolumn{5}{|l|}{ Stigmatisierung } \\
\hline Angst, Kollegen/Freunden von der Therapie zu erzählen & $12(29,3 \%)$ & 1,5 & $1-3$ & 0,7 \\
\hline
\end{tabular}

\section{Negative Effekte}

Die Befragten gaben im Mittel 11,2 negative Effekte an ( $\mathrm{SD}=6,6$, Range: $1-28$ ), die sie subjektiv auf die tagesklinische DBT-Behandlung zurückführten. Diese sind anhand des INEP in Tabelle 2 einzeln und in Bereiche geordnet aufgeführt.

Bezüglich therapeutischer Fehlhandlungen wurde einmalig angegeben, dass die Schweigepflicht gebrochen worden sei. Nicht berichtet wurden sexuelle oder körperliche Übergriffe.

Am häufigsten waren im INEP die Bereiche intrapersonelle Symptomatik und therapeutische Beziehung als negativ erlebt worden. Die häufigsten subjektiv erlebten, negativen Einzeleffekte aufgrund der Therapie nach beendeter tagesklinischer DBT sind zum einen "längere Phasen in schlechter psychischer Verfassung" als auch "Schwierigkeiten, Entscheidungen allein zu treffen" sowie "vermehrte finanzielle Sorgen" zu haben.

\section{Mögliche Einflussfaktoren}

Aufgrund der wenigen befragten Männer war eine Assoziationsanalyse des Geschlechts zum Gesamt-INEP nicht durchführbar. Hinsichtlich des Schulabschlusses und der Mindestanzahl an Suizidversuchen war eine As- soziation statistisch durchführbar, aber nicht nachweisbar (Tabelle 1). Ebenfalls ist anhand der vorgestellten Daten kein Zusammenhang zu negativen Effekten und der Ausprägung der BPS anhand der DSM-IV-Anzahl feststellbar. Auch für die Länge der Therapie, die im Durchschnitt und auch überwiegend bei 83 Tagen (etwa 12 Wochen) lag, konnte keine Assoziation beschrieben werden. Der Zeitraum seit Therapieende bis zur Befragung war im Durchschnitt mit 21,6 Monaten recht lang und zeigte eine Spannweite von 3 bis 51 Monaten. Dieser zeigte aber statistisch keinen Zusammenhang mit erinnerten negativen Effekten.

Jedoch kann ein statistischer Zusammenhang als stochastische Korrelation und auch jeweils als lineare Regression zwischen INEP-Gesamt und dem Alter $(p<$ $0,01)$ sowie der BSL-95 $(p<0,01)$ bzw. BDI $(p<0,01)$ zum Befragungszeitpunkt hergeleitet werden (Tabelle 1).

Daten aus einer früheren Studie der gleichen Patientenstichprobe [Richter et al., 2014a] wurden herangezogen, um mögliche Verschlechterungen bzw. Verbesserungen der Symptome über verschiedene Zeitpunkte mit dem INEP zu assoziieren. Die jeweiligen Differenzen der BSL-95 zum Therapiestart (T1), Therapieende (T2) und dem Befragungszeitpunkt (T3) wurden hierfür von T1 zu 
Tabelle 3. Assoziation zur Symptomänderung über drei Zeitpunkte mit dem INEP $(\mathrm{T} 1=$ Therapiestart, $\mathrm{T} 2=$ Therapieende, $\mathrm{T} 3$ = Befragungszeitpunkt)

\begin{tabular}{llrrl}
\hline & $n=41(100 \%)$ & M & SD & $\begin{array}{l}\text { Korrelation mit } \\
\text { INEP-Gesamtanzahl }\end{array}$ \\
\hline Differenz BSL-95 (T1) minus BSL-95 (T2) & $29(70,7 \%)$ & 67,1 & 59,3 & $\begin{array}{r}r=-0,02 \\
r=-0,42^{*}\end{array}$ \\
Differenz BSL-95 (T2) minus BSL-95 (T3) & $32(78,0 \%)$ & $-27,9$ & 79,4 & $r=-0.06$ \\
\hline
\end{tabular}

M, Mittelwert; SD, Standardabweichung; $r$, Korrelation nach Pearson. ${ }^{*} p<0.05$.

T2 anhand der BSL-95 bezüglich negativer Effekte (Tabelle 3) erfasst. Jedoch werden bei Symptomverschlechterung anhand der BSL-95 seit Therapieende bis zum Befragungszeitpunkt mehr negative Effekte angegeben, die korreliert und in einem (linearen) Zusammenhang beschrieben werden können (Differenz BSL-95 T2 minus BSL-95 T3, p < 0,05). Dennoch geben zehn Befragte negative Effekte der DBT auch an $(\mathrm{M}=9 ; \mathrm{SD}=8,7$; Range: 1-28), wenn es zum Befragungszeitpunkt zu keiner Verschlechterung bzw. sogar zu einer Verbesserung ihrer Symptomatik anhand der BSL-95 im Vergleich zum Therapieende kam. Ein statistischer Zusammenhang der Differenz BSL-95 (T1) minus BSL-95 (B3) mit negativen Effekten konnte nicht gefunden werden.

\section{Diskussion}

Die Daten zeigen, dass BPS-Patienten etwa zwei Jahre nach Entlassung aus einer teilstationären Behandlung auf dem INEP über eine erhebliche Rate an unerwünschten Ereignissen klagen. 97,6\% berichten von Verschlechterung ihres Befindens durch die teilstationäre Behandlung, $87,8 \%$ erinnern sich an Probleme in der therapeutischen Beziehung, 43,9\% klagen über Verschlechterungen in der Beziehung, 70,7\% über finanzielle Probleme in Folge der tagesklinischen Behandlung und 29,3\% über Stigmatisierungserleben in Folge der Behandlung. Dies sind beeindruckende Raten von Negativberichten. Die Frage ist, was bei einer retrospektiven Befragung nach langem Zwischenzeitraum mit dem INEP erfasst wird.

Die Antworten im INEP können sich aufgrund dieser Zeitspanne verändert haben. Möglicherweise sähen die Ergebnisse direkt nach Ende der Therapie oder begleitend zu dieser mit dem gleichen Fragebogen anders aus. Auch ist nicht bekannt, ob im Zeitraum zwischen dem Ende der Therapie und der Befragung Ereignisse stattfanden, die dazu geführt haben könnten, die Therapie jetzt in dieser Weise zu bewerten. Es zeigt sich allerdings kein Zusammenhang zwischen der Zeit seit Therapieende und Befragungszeitpunkt. Es bleibt auch offen, worauf sich die Angaben im Detail beziehen, da es sich um eine komplexe teilstationäre Behandlung handelte, bei der DBT nur ein Behandlungsteil war.

Die Ergebnisse der Erhebung zeigen im Vergleich zu Abeling et al. [2018] und Grüneberger et al. [2017], dass bei einem speziellen Störungsbild hier am Beispiel der BPS bestimmte negative Werte (z.B. den Bereich intrapersonelle Symptomatik betreffend, wie "längere Phasen in schlechter psychischer Verfassung") stärker von Patientenseite angegeben werden. Dies muss nicht notwendigerweise am Therapiesetting liegen, sondern kann auch durch dysfunktionale Denk- und Verhaltensweisen der BPS [Stiglmayr, 2003] und den chronischen Verlauf der Persönlichkeitsstörung bedingt sein (auch wenn die Symptomausprägung nach Therapie reduziert werden konnte). Hierfür wäre eine weiterführende, dann kontrollierte Studie notwendig. Kontrollierte Studien liegen jedoch bisher in rarer Anzahl vor und beschreiben negative Effekte oder auch nur Nebenwirkungsklagen von Psychotherapie zumeist nur allgemein [Jonsson et al., 2014; Vaughan et al., 2014; Schneibel et al., 2017].

Die subjektiv erlebten Schwierigkeiten in der therapeutischen Beziehung könnten Folge spezieller Face-toFace-Kontaktproblematiken der BPS sein [Bilek et al., 2017]. Das erhöhte "Gefühl, dass der Therapeut sich über den Patienten lustig macht", kann eben durch diese BPSspezifisch veränderte Wahrnehmungsschwierigkeit gesehen werden, die diese Patienten in zwischenmenschlichen Kontakten erleben [Izurieta Hidalgo et al., 2016; Bertsch et al., 2018]. Als Alternativhypothese wäre auch anzunehmen, dass gerade in dieser untersuchten Tagesklinik Verletztheit durch Therapeutenaussagen verstärkt vorkommt, was nicht zu hoffen ist, aber auch nicht ausgeschlossen werden kann. Nur eine Multicenter-Studie könnte dies sicher klären.

Im Vergleich zu den beiden anderen INEP-Studien von Abeling et al. [2018] und Grüneberger et al. [2017] ist ersichtlich, dass diese DBT-Studienkohorte einen höheren Anteil an "längeren Phasen bei schlechter psychischer Gesundheit" und "mehr Suizidgedanken zum ersten Mal während der Therapie" beschreibt. Dies könnte erstens mit der nur ambulanten, wahrscheinlich weniger 
akuten Studienpopulation bei Grüneberger et al. [2017] und zweitens aufgrund einer nicht nach Diagnosen differenzierten Studienpopulation erklärt werden. In der aktuellen Umfrage fanden ausschließlich Patienten mit BPS Eingang, die möglicherweise symptombelasteter sind (sonst wäre eine rein ambulante Therapie ausreichend gewesen) und vor allem chronische Suizidgedanken als Symptom in die Therapie der BPS einbringen.

Andererseits ist gerade im Kontext der BPS bekannt, dass der Umgang und die Bedeutung negativer Effekte und Störungen der therapeutischen Beziehung Einfluss auf das Outcome einer Therapie haben können [Boritz et al., 2018]. Auch wissen Kliniker um Phasen großer Instabilität und vorübergehender Belastung durch Exposition, die aber langfristig positive Veränderungen bewirken [Olthof et al., 2020].

DBT in der Tagesklinik ist vor allem Gruppen- und in geringerem Umfang Einzeltherapie. Auch Linden et al. [2015] konnten zeigen, dass mit belastenden und damit unerwünschten Erfahrungen während Gruppentherapien insbesondere zu rechnen ist.

Unter Gesichtspunkten der Validität der berichteten Klagen ist auch von Interesse, dass ca. 70\% der Studienpopulation über größere finanzielle und rechtliche Nachteile berichten. Wie das eine Nebenwirkung der teilstationären Behandlung sein könnte, ist nicht vorstellbar. Aufgrund der vollständigen Übernahme der Behandlungskosten durch die gesetzliche Krankenversicherung in Deutschland für diese tagesklinische DBT - und die befragten Patientinnen und Patienten waren ausschließlich so versichert - sind keine neuen finanziellen Schulden durch diese Therapie entstanden. Wenn es sich um Nebenwirkungen der Therapie handeln sollte, könnten es durch die Therapie neu wahrgenommene Sorgen über die bestehende finanzielle Situation sein, was aber auch nicht als unerwünschtes Ereignis zu bewerten wäre. Es ist ja gerade bekannt, dass etwa vier von fünf BPS-Patienten keine bezahlte Beschäftigung haben [Bohus und Kröger, 2011].

Der beschriebene negative Effekt, sich "als Mensch zum Negativen" verändert zu haben, könnte mit einem geringen Selbstwert von BPS-Patienten erklärbar sein. Schon ältere Publikationen bringen negative Ergebnisse bei Psychotherapie dieser Patientenpopulation hiermit in Verbindung [Kernberg et al., 1972; Stone, 1990]. Die hohe Rate an Klagen könnte also eine besonders selbstwertdienliche Verzerrung von BPS-Patienten in der Zeit nach der Therapie darstellen, um eigene Misserfolge (hier erhoffte, aber nicht realisierte Erfolgswünsche nach der Therapie) eher äußeren Ursachen (hier der Therapie) zuzuschreiben [Miller und Ross, 1975].

Die von etwa jedem dritten beschriebene Abhängigkeit vom Therapeuten und die Reduzierung unabhängiger Entscheidungen sind sicherlich als unerwünschte
Effekte zu betrachten, wenn sie mit dem tagesklinischen Aufenthalt zusammenhängen würden [Freyberger et al., 2013]. Rückfälle bzw. die Sorge, diese könnten auftreten, wenn die Psychotherapie beendet wird, werden auch bei anderen Autoren z.B. für Gruppentherapie benannt [Nutt und Sharpe, 2008].

Zusammenfassend stellt sich die Frage, wie Ergebnisse auf dem INEP-Selbstratingfragebogen zu verstehen und $\mathrm{zu}$ interpretieren sind. Das INEP wurde zur Erfassung von Nebenwirkungen entwickelt. Die vorliegenden Daten sprechen jedoch dafür, dass die Validität der so erfassten Berichte in dieser Befragung zu hinterfragen ist. Wie sollen sich Patienten nach durchschnittlich zwei Jahren nach Therapieende an konkrete therapiebedingte Belastungen erinnern? Bei der Interpretation der Ergebnisse ist zudem von großer Bedeutung, dass offenbar der aktuelle psychische Befund die retrospektive Beurteilung von negativen Effekten beeinflusst. Dies schließt nicht aus, dass kränkere Patienten mehr Nebenwirkungen erleben. Bei der Art der Klagen spricht aber viel dafür, dass die Antworten auf dem INEP weniger etwas über Nebenwirkungen einer zwei Jahre zurückliegenden teilstationären Behandlung sagen, als vielmehr ein undifferenziertes Klage- und Antwortverhalten der Patienten wiederspiegeln. Das INEP ist als Hinweisinstrument zu verstehen, um patientenbezogene Sorgen und Befürchtungen im klinischen Alltag zu thematisieren und gegebenenfalls zu korrigieren. Die alleinige Anwendung des INEP zur Erfassung von Nebenwirkungen ist mit erheblichen Unschärfen verbunden.

Diese Untersuchung soll der Sensibilisierung zum Thema dienen. Die Beachtung negativer Effekte von Psychotherapie ist unverzichtbar, die Erfassung stellt jedoch erhebliche methodische Probleme. Standardisierte Protokolle können dennoch Patienten und Therapeuten hierfür sensibilisieren [Czaja et al., 2006].

\section{Danksagung}

Mein Dank gilt Sandra Schild und Genia Juschkewitsch von der Psychologischen Universität Berlin, die die Datenerhebung im Rahmen ihrer Masterarbeit übernommen haben. Für die Beratung im Vorfeld der Studie bedanke ich mich ausdrücklich bei Prof. E.L. Brakemeier. Für die wissenschaftliche Verwendung des INEPFragebogens danke ich der Forschungsgruppe Ladwig \& Rief \& Nestoriuc. Ohne die engagierte Arbeit des gesamten Therapeutenteams der Tagesklinik 1 im Vivantes Wenckebach-Krankenhaus wäre diese Arbeit nicht möglich gewesen.

\section{Conflict of Interest Statement}

Der Autor ist Facharzt für Psychiatrie und Psychotherapie mit Schwerpunkt Verhaltenstherapie und zertifizierter DBT-Therapeut nach den Richtlinien des Dachverbandes Dialektisch Behaviorale Therapie e.V. Er war als Vivantes-Mitarbeiter zum Zeitpunkt der 
Erhebung oberärztlich für die Etablierung des Konzeptes der benannten Tagesklinik zuständig. Finanzielle Zuwendungen durch Pharmafirmen oder andere Personen/Institutionen, die in irgendeiner Weise diese Arbeit beeinflussen können, existieren ebenfalls nicht. Der Autor erklärt, dass kein Interessenkonflikt besteht.

\section{Finanzielle Unterstützung}

Die Studie mit Veröffentlichung wurde finanziell nicht von Dritten unterstützt. Die Vivantes GmbH trug Bürokosten über Sachmittel.

\section{Literatur}

Abeling B, Müller A, Stephan M, Pollmann I, de Zwaan M. [Negative Effects of Psychotherapy: Prevalence and Correlates in a Clinical Sample]. Psychother Psychosom Med Psychol. 2018 Sep;68(9-10):428-36.

Bertsch K, Hillmann K, Herpertz SC. Behavioral and Neurobiological Correlates of Disturbed Emotion Processing in Borderline Personality Disorder. Psychopathology. 2018;51(2):7682.

Bilek E, Stößel G, Schäfer A, Clement L, Ruf M, Robnik L, et al. State-Dependent Cross-Brain Information Flow in Borderline Personality Disorder. JAMA Psychiatry. 2017 Sep;74(9): 949-57.

Bohus M, Kröger C. Psychopathologie und Psychotherapie der Borderline-Persönlichkeitsstörung: Zum gegenwärtigen Stand der Forschung. Nervenarzt. 2011 Jan;82(1):1624.

Bohus M, Limberger MF, Frank U, Chapman AL, Kühler T, Stieglitz RD. Psychometric properties of the Borderline Symptom List (BSL). Psychopathology. 2007;40(2):126-32.

Bohus M. Borderline-Störung. Göttingen: Hogrefe; 2019. https://doi.org/10.1026/02853000 .

Boritz T, Barnhart R, Eubanks CF, McMain S. Alliance Rupture and Resolution in Dialectical Behavior Therapy for Borderline Personality Disorder. J Pers Disord. 2018 Jan;32 Suppl: 115-28.

Brakemeier EL, Breger V, Spitzer C. Nebenwirkungen von ambulanter und stationärer Psychotherapie. Verhaltensther Psychosoz Prax. 2012;44:489-510.

Carpenter RW, Trull TJ. Components of emotion dysregulation in borderline personality disorder: a review. Curr Psychiatry Rep. 2013 Jan; 15(1):335.

Cristea IA, Gentili C, Cotet CD, Palomba D, Barbui C, Cuijpers P. Efficacy of Psychotherapies for Borderline Personality Disorder: A Systematic Review and Meta-analysis. JAMA Psychiatry. 2017 Apr;74(4):319-28.

Crowell SE, Beauchaine TP, Linehan MM. A biosocial developmental model of borderline personality: elaborating and extending Linehan's theory. Psychol Bull. 2009 May;135(3): 495-510.

Czaja SJ, Schulz R, Belle SH, Burgio LD, Armstrong N, Gitlin LN, et al. Data and safety monitoring in social behavioral intervention trials: the REACH II experience. Clin Trials. 2006;3(2):107-18.

Duggan C, Parry G, McMurran M, Davidson K, Dennis J. The recording of adverse events from psychological treatments in clinical trials: evidence from a review of NIHR-funded trials. Trials. 2014 Aug;15(1):335.
Ellison WD, Rosenstein LK, Morgan TA, Zimmerman M. Community and Clinical Epidemiology of Borderline Personality Disorder. Psychiatr Clin North Am. 2018 Dec;41(4): 561-73.

Freyberger H, Haupt ML, Hellweg R, Hilgers M, Hillebrand V, Jakl B, et al. Risiken und Nebenwirkungen von Psychotherapie. Erfassung, Bewältigung, Risikovermeidung. Berlin: MWV Medizinisch Wissenschaftliche Verlagsgesellschaft; 2013.

Grüneberger A, Einsle F, Hoyer J, Strauß B, Linden M, Härtling S. [Subjective Adverse Effects during Outpatient CBT: Associations to $\mathrm{Pa}-$ tient and Therapist Variables and to the Therapeutic Alliance]. Psychother Psychosom Med Psychol. 2017 Aug;67(8):338-44.

Hautzinger M, Bailer M, Worall H. BDI Beck-Depressions-Inventar Testhandbuch (German Version). 2nd ed. Bern: Verlag Hans Huber; 1995.

Holsting AF, Pedersen HF, Rask MT, Frostholm L, Schröder A. Is psychotherapy for functional somatic syndromes harmful? A mixed methods study on negative effects. J Psychosom Res. 2017 Jul;98:113-21.

Hopf H. Praxishandbuch Tageskliniken - Teilstationäre Behandlung in Psychiatrie und Psychosomatik. Elsevier; 2018.

Izurieta Hidalgo NA, Oelkers-Ax R, Nagy K, Mancke F, Bohus M, Herpertz SC, et al. Time course of facial emotion processing in women with borderline personality disorder: an ERP study. J Psychiatry Neurosci. 2016 Jan;41(1): $16-26$.

Jacobi F. Misserfolgsforschung in der Verhaltens therapie. Lengerich. Pabst; 2001.

Jonsson U, Alaie I, Parling T, Arnberg FK. Reporting of harms in randomized controlled trials of psychological interventions for mental and behavioral disorders: a review of current practice. Contemp Clin Trials. 2014 May; 38(1):1-8.

Kernberg OF, Coyne L, Horwitz L, Appelbaum A, Burstein ED. Psychotherapy and psychoanalysis. The application of facet theory and the multidimensional scalogram analysis to the quantitative data of the psychotherapy research project. Bull Menninger Clin. 1972 Jan-Mar;36(1):87-275.

Kienast T, Stoffers J, Bermpohl F, Lieb K. Borderline personality disorder and comorbid addiction: epidemiology and treatment. Dtsch Arztebl Int. 2014 Apr;111(16):280-6.

Kliem S, Kröger C, Kosfelder J. Dialectical behavior therapy for borderline personality disorder: a meta-analysis using mixed-effects modeling. J Consult Clin Psychol. 2010 Dec;78(6): 936-51.
Ladwig I, Rief W, Nestoriuc Y. Welche Risiken und Nebenwirkungen hat Psychotherapie? Entwicklung des Inventars zur Erfassung Negativer Effekte von Psychotherapie (INEP) [What Are the Risks and Side Effects of Psychotherapy? - Development of an Inventory for the Assessment of Negative Effects of Psychotherapy (INEP)]. Verhaltenstherapie. 2014;24(4):252-63.

Levant R, Barlow D, David K, Hagglund K, Hollon S, Johnson J, et al.; APA Presidential Task Force on Evidence-Based Practice. Evidencebased practice in psychology. Am Psychol. 2006 May-Jun;61(4):271-85.

Lieb K, Völlm B, Rücker G, Timmer A, Stoffers JM. Pharmacotherapy for borderline personality disorder: cochrane systematic review of randomised trials. Br J Psychiatry. 2010 Jan; 196(1):4-12.

Linden M, Walter M, Fritz K, Muschalla B. [Undesired treatment effects in behavior group therapy: frequency and spectrum]. Nervenarzt. 2015 Nov;86(11):1371-82.

Linden M. How to define, find and classify side effects in psychotherapy: from unwanted events to adverse treatment reactions. Clin Psychol Psychother. 2013 Jul-Aug;20(4):28696.

Linden M. Psychosomatic inpatient rehabilitation: the German model. Psychother Psychosom. 2014;83(4):205-12.

Linehan M. Handbuch der Dialektisch-Behavioralen Therapie (DBT), Skills Training Manual \& Arbeitsbuch. München, CIP-Medien, 2016.

Lynch TR, Trost WT, Salsman N, Linehan MM. Dialectical behavior therapy for borderline personality disorder. Annu Rev Clin Psychol. 2007;3(1):181-205.

McCay E, Carter C, Aiello A, Quesnel S, Howes C, Johansson B. Toward Treatment Integrity: Developing an Approach to Measure the Treatment Integrity of a Dialectical Behavior Therapy Intervention With Homeless Youth in the Community. Arch Psychiatr Nurs. 2016 Oct;30(5):568-74.

Miller DT, Ross M. Self-serving biases in the attribution of causality: fact or fiction? Psychol Bull. 1975;82(2):213-25.

Mohr DC. Negative Outcome in Psychotherapy: A Critical Review. Clin Psychol Sci Pract. 1995;2(1): 1-27.

Nutt DJ, Sharpe M. Uncritical positive regard? Issues in the efficacy and safety of psychotherapy. J Psychopharmacol. 2008 Jan;22(1):3-6.

Olthof M, Hasselman F, Strunk G, Aas B, Schiepek G, Lichtwarck-Aschoff A. Destabilization in self-ratings of the psychotherapeutic process is associated with better treatment outcome in patients with mood disorders. Psychother Res. 2020 Apr;30(4):520-31. 
Parker G, Fletcher K, Berk M, Paterson A. Development of a measure quantifying adverse psychotherapeutic ingredients: the Experiences of Therapy Questionnaire (ETQ). Psychiatry Res. 2013 Apr;206(2-3):293-301.

Parry GD, Crawford MJ, Duggan C. Iatrogenic harm from psychological therapies - time to move on. Br J Psychiatry. 2016 Mar;208(3): 210-2.

Pasieczny N, Connor J. The effectiveness of dialectical behaviour therapy in routine public mental health settings: an Australian controlled trial. Behav Res Ther. 2011 Jan;49(1) 4-10.

Petry NM, Roll JM, Rounsaville BJ, Ball SA, Stitzer M, Peirce JM, et al. Serious adverse events in randomized psychosocial treatment studies: safety or arbitrary edicts? J Consult Clin Psychol. 2008 Dec;76(6):1076-82.

Priebe S, Bhatti N, Barnicot K, Bremner S, Gaglia A, Katsakou C, et al. Effectiveness and costeffectiveness of dialectical behaviour therapy for self-harming patients with personality disorder: a pragmatic randomised controlled trial. Psychother Psychosom. 2012;81(6):35665.

Richter C, Heinemann B, Kehn M, Steinacher B. Effektivität der Dialektisch-Behavioralen Therapie (DBT) in der tagesklinischen Behandlung der Borderline-Persönlichkeitsstörung - Bedeutung von Medikation und Behandlungskosten [Effectiveness of Dialectical Behavior Therapy (DBT) in an outpatient clinic for borderline personality disorders - impact of medication use and treatment costs]. Psychiatr Prax. 2014a Apr;41(3): 148-52.
Richter C, Steinacher B, zum Eschenhoff A, Bermpohl F. Stationäre und teilstationäre DBT-Angebote für Patienten mit BorderlinePersönlichkeitsstörung - Ergebnisse einer deutschlandweiten Umfrage. Verhaltenstherapie. $2014 b ; 24(4): 265-71$.

Richter C. Tagesklinische dialektisch-behaviorale Therapie: Eine Verlaufsuntersuchung nach Entlassung. Gibt es Prädiktoren für eine Verschlechterung? Verhaltenstherapie. 2019; 29(1):1-9.

Sansone RA, Wiederman MW. The abuse of prescription medications: borderline personality patients in psychiatric versus non-psychiatric settings. Int J Psychiatry Med. 2009;39(2): 147-54.

Schmahl C, Herpertz SC, Bertsch K, Ende G, Flor $\mathrm{H}$, Kirsch P, et al. Mechanisms of disturbed emotion processing and social interaction in borderline personality disorder: state of knowledge and research agenda of the German Clinical Research Unit. Borderline Personal Disord Emot Dysregul. 2014 Sep;1(1): 12.

Schneibel R, Wilbertz G, Scholz C, Becker M, Brakemeier EL, Bschor $\mathrm{T}$, et al. Adverse events of group psychotherapy in the in-patient setting - results of a naturalistic trial. Acta Psychiatr Scand. 2017 Sep;136(3):24758.

Scott J, Young AH. Psychotherapies should be assessed for both benefit and harm. Br J Psychiatry. 2016 Mar;208(3):208-9.

Signer S, Estermann Jansen R, Sachse R, Caspar F, Kramer U. Social interaction patterns, therapist responsiveness, and outcome in treatments for borderline personality disorder. Psychol Psychother. 2019 Oct 4:e12254.
Stiglmayr C, Stecher-Mohr J, Wagner T, Meißner J, Spretz D, Steffens C, et al. Effectiveness of dialectic behavioral therapy in routine outpatient care: the Berlin Borderline Study. Borderline Personal Disord Emot Dysregul. 2014 Dec;1(1):20.

Stiglmayr C. Spannung und Dissoziation bei der Borderline-Persönlichkeitsstörung. Frankfurt/M. Berlin, Bern, Bruxelles, New York, Oxford, Wien: Verlag Peter Lang; 2003.

Stoffers JM, Völlm BA, Rücker G, Timmer A, Huband N, Lieb K. Psychological therapies for people with borderline personality disorder. Cochrane Database Syst Rev. 2012 Aug;(8):CD005652.

Stone MH. Treatment of borderline patients: a pragmatic approach. Psychiatr Clin North Am. 1990 Jun;13(2):265-85.

Suh C, Strupp H, O'Malley S. The Vanderbilt process measures: The Psychotherapy Process Scale (VPPS) and the Negative Indicators Scale (VNIS). New York: Guilford Press; 1986.

van den Bosch LM, Sinnaeve R, Hakkaart-van Roijen L, van Furth EF. Efficacy and cost-effectiveness of an experimental short-term inpatient Dialectical Behavior Therapy (DBT) program: study protocol for a randomized controlled trial. Trials. 2014 May;15(1):152.

Vaughan B, Goldstein MH, Alikakos M, Cohen LJ, Serby MJ. Frequency of reporting of adverse events in randomized controlled trials of psychotherapy vs. psychopharmacotherapy. Compr Psychiatry. 2014 May;55(4):84955. 